FERMILAB-SLIDES-20-016-QIS

This document has been authored by Fermi Research Alliance, LLC under Contract No. DE-AC02-07CH11359 with the U.S. Department of Energy, Office of Science, Office of High Energy Physics.

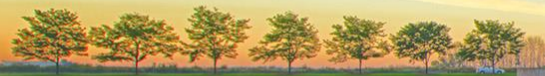

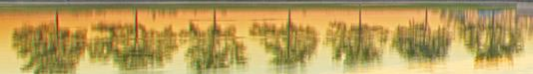

\section{Quantum Computing for Neutrino-nucleus Scattering with NISQ Devices}

Andy C. Y. Li (Fermilab Quantum Institute) Quantum Computing User Forum at ORNL 21 April 2020
Alessandro Roggero (UW) Andy C. Y. Li (Fermilab) Joseph Carlson (LANL) Rajan Gupta (LANL) Gabriel N. Perdue (Fermilab) preprint: arXiv: 1911.06368 (accepted by Physical Review D) 


\section{Neutrino-nucleus scattering}

- Accelerator Neutrino Experiments, e.g. DUNE

- Simulate scattering cross sections to predict detector efficiency and backgrounds

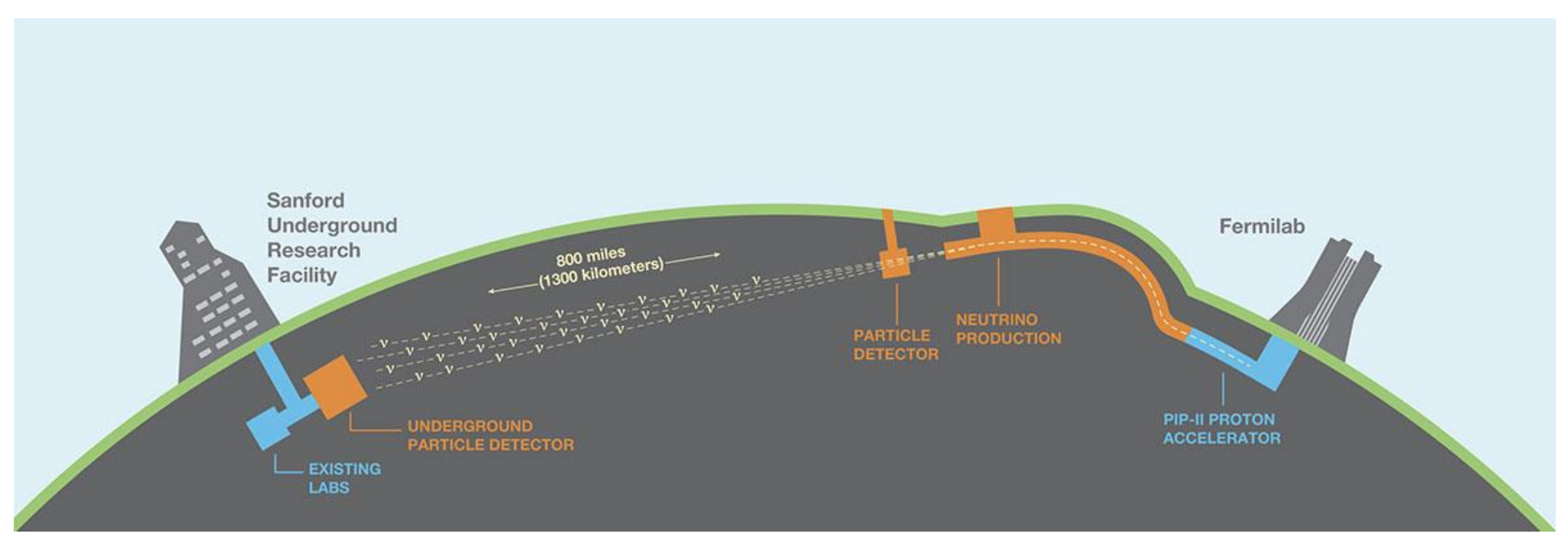




\section{Simulate response function and cross sections}

- Dynamical linear response function

$$
\begin{gathered}
S(\omega, \hat{O})=\sum_{v}\left|\left\langle\phi_{v}|\hat{O}| \phi_{0}\right\rangle\right|^{2} \delta\left(E_{v}-E_{0}-\omega\right)=\int d t\left\langle\phi_{0}\left|\hat{O}^{\dagger} e^{-i\left(\widehat{H}-E_{0}-\omega\right) t} \hat{O}\right| \phi_{0}\right\rangle \\
\text { Nuclei: } \widehat{H}\left|\phi_{v}\right\rangle=E_{v}\left|\phi_{v}\right\rangle \quad \text { ground state: }\left|\phi_{0}\right\rangle
\end{gathered}
$$

- $S(\omega, \hat{O}) \rightarrow$ inclusive cross sections

- Sample the final nuclei state $\left|\phi_{v}\right\rangle \rightarrow$ semi-exclusive cross sections

- Quantum advantage: bigger nuclei, wide range of kinematics 


\section{Starting point: pionless effective field theory}

$$
\begin{aligned}
H & =2 D t A-t \sum_{f=1}^{N_{f}} \sum_{\langle i, j\rangle}^{M}\left[c_{i, f}^{\dagger} c_{j, f}+c_{i, f}^{\dagger} c_{j, f}\right] \\
& +\frac{1}{2} C_{0} \sum_{f \neq f^{\prime}}^{N_{f}} \sum_{i=1}^{M} n_{i, f} n_{i, f^{\prime}} \\
& +\frac{D_{0}}{6} \sum_{f \neq f^{\prime} \neq f^{\prime \prime}}^{N_{f}} \sum_{i=1}^{M} n_{i, f} n_{i, f^{\prime}} n_{i, f^{\prime \prime}}
\end{aligned}
$$

Kinetic energy

Attractive 2-body contact interaction $\left(C_{0}<0\right)$

Repulsive 3-body interaction $\left(D_{0}>0\right)$ to avoid collapse into deeply bound state

- Approximately reproduce binding of 3 and 4 nucleons

[Phys. Lett. B 772 839-848 (2017), PRL 124143402 (2020)]

- Simple model for initial study and quantum resource estimation

- Future: need interactions involving virtual pions for accurate prediction 


\section{Dynamic linear response quantum algorithm}

$$
\begin{aligned}
S(\omega, \hat{O}) & =\sum_{v}\left|\left\langle\phi_{v}|\hat{O}| \phi_{0}\right\rangle\right|^{2} \delta\left(E_{v}-E_{0}-\omega\right) \\
& =\sum_{v}\left|\left\langle\phi_{v} \mid \psi_{\hat{O}}\right\rangle\right|^{2} \delta\left(E_{v}-E_{0}-\omega\right)\left\langle\phi_{0}\left|\hat{O}^{\dagger} \hat{O}\right| \phi_{0}\right\rangle
\end{aligned}
$$

Prob. of $\left|\psi_{\hat{o}}\right\rangle$ in eigenbasis $\left|\phi_{v}\right\rangle \rightarrow$ QPE

Ground state meas.

Qubit encoding:represent the system by qubits

\section{Complexity}

2. State preparation: $\left|\psi_{\hat{o}}\right\rangle$
Dynamic linear response quantum algorithm Alessandro Roggero ${ }^{*}$ and Joseph Carlson ${ }^{\dagger}$

Theoretical Division, Los Alamos National Laboratory, Los Alamos, New Mexico 87545, USA

(Q) (Received 15 May 2018; published 13 September 2019)

3. Quantum phase estimation of $\left|\psi_{\hat{o}}\right\rangle$ with $\widehat{U}=e^{i\left(\widehat{H}-E_{0}\right)}$

4. Measure ancilla qubits: probability distribution $\rightarrow S(\omega, \hat{O})$ (nuclei state by measuring the encoding qubits)

Superposition $\quad$ Controlled $U$ Operations

$$
\left|\psi_{\hat{O}}\right\rangle=\frac{\hat{O}\left|\phi_{0}\right\rangle}{\sqrt{\left\langle\phi_{0}\left|\hat{O}^{\dagger} \hat{O}\right| \phi_{0}\right\rangle}}
$$
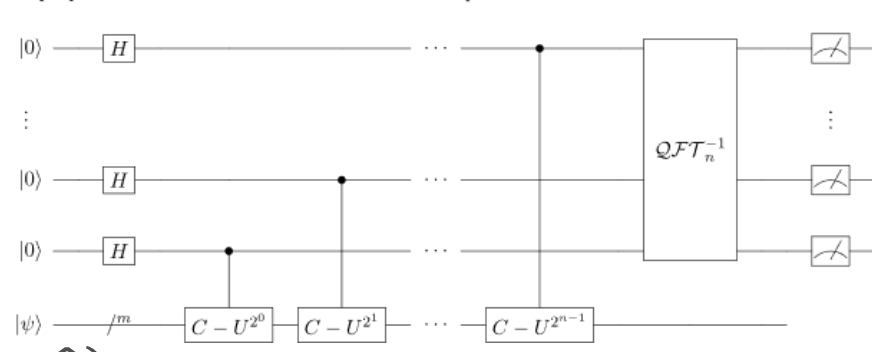


\section{Qubit encoding efficiency}

- Nucleons (fermions) $\rightarrow$ qubits

- General mapping: Jordan-Wigner, Bravyi-Kitaev [1], etc.

$$
\begin{aligned}
H & =2 D t A-t \sum_{f=1}^{N_{f}} \sum_{\langle i, j\rangle}^{M}\left[c_{i, f}^{\dagger} c_{j, f}+c_{i, f}^{\dagger} c_{j, f}\right] \\
& +\frac{1}{2} C_{0} \sum_{f \neq f^{\prime}}^{N_{f}} \sum_{i=1}^{M} n_{i, f} n_{i, f^{\prime}}
\end{aligned}
$$

- Special case of fixed nucleons: lattice-location encoding nucleon 1: $|1\rangle_{N 1}=|0\rangle_{q 0}|0\rangle_{q 1},|2\rangle_{N 1}=|0\rangle_{q 0}|1\rangle_{q 1}, \ldots$ nucleon 2: $|1\rangle_{N 2}=|0\rangle_{q 2}|0\rangle_{q 3},|2\rangle_{N 2}=|0\rangle_{q 2}|1\rangle_{q 3}, \ldots$

- Efficiency: $A$ nucleons on a lattice with $M$ sites and $N_{f}$ fermion mode per site

JW, BK

: $N_{f} \times M$ qubits

Lattice-location

: $A \log _{2} M$ qubits

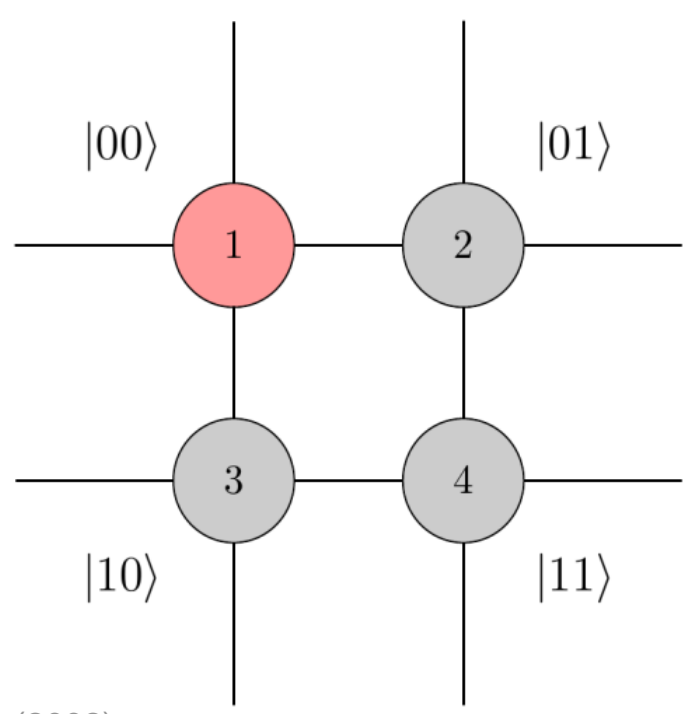




\section{Quantum phase estimation}

- QFT and Control- $U$ circuits $\widehat{U}=e^{i\left(\widehat{H}-E_{0}\right)}:$ system propagator

- QFT: gate cost $=O\left(N^{2}\right)$ $N$ : number of ancilla qubits

|0) $-H$

$|0\rangle-H$

$|0\rangle-H$

$|\psi\rangle$

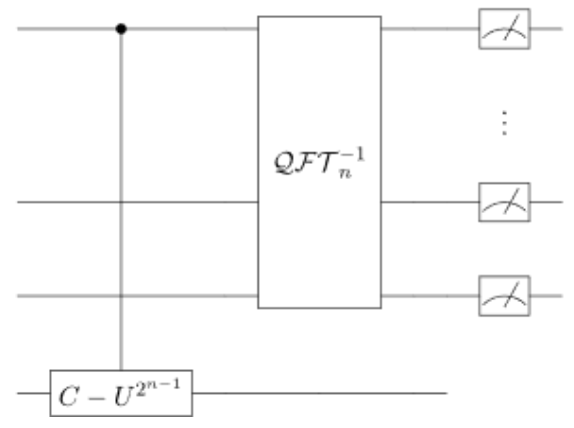

- $U$ circuits: Trotter decompositions

- $U_{1}(\tau)=e^{-i \tau K} e^{-i \tau V}$

$-U_{2}^{K+V}(\tau)=e^{-i \tau K / 2} e^{-i \tau V} e^{-i \tau K / 2}$

$-U_{2}^{V+K}(\tau)=e^{-i \tau V / 2} e^{-i \tau K} e^{-i \tau V / 2}$

- Control- $U$ circuits: replace gates by their $H=2 D t A-t \sum_{f=1}^{N_{f}} \sum_{\langle i, j\rangle}^{M}\left[c_{i, f}^{\dagger} c_{j, f}+c_{i, f}^{\dagger} c_{j, f}\right]$
$+\frac{1}{2} C_{0} \sum_{f \neq f^{\prime}}^{N_{f}} \sum_{i=1}^{M} n_{i, f} n_{i, f^{\prime}}$
$+\frac{D_{0}}{6} \sum_{f \neq f^{\prime} \neq f^{\prime \prime}}^{N_{f}} \sum_{i=1}^{M} n_{i, f} n_{i, f^{\prime}} n_{i, f^{\prime \prime}}$, K: kinetic energy $V$ : potential energy Diagonal in qubit basis after JW controlled version 


\section{Gate counts of quantum phase estimation}

- Gate counts based on 2 gates

- CNOT: control-not, two-qubit gate

- $R_{Z}$ : rotation-Z, single-qubit gate

- Quadratic decomposition: favorable

- Gate counts $\rightarrow \sim 10^{10}$

- Final 99\% fidelity: $1-e^{\frac{\ln 0.99}{10^{10}}}$

$\rightarrow \sim 10^{-12}$ gate error rate

- Need error-corrected qubits for full linear response algorithm simulating realistic model

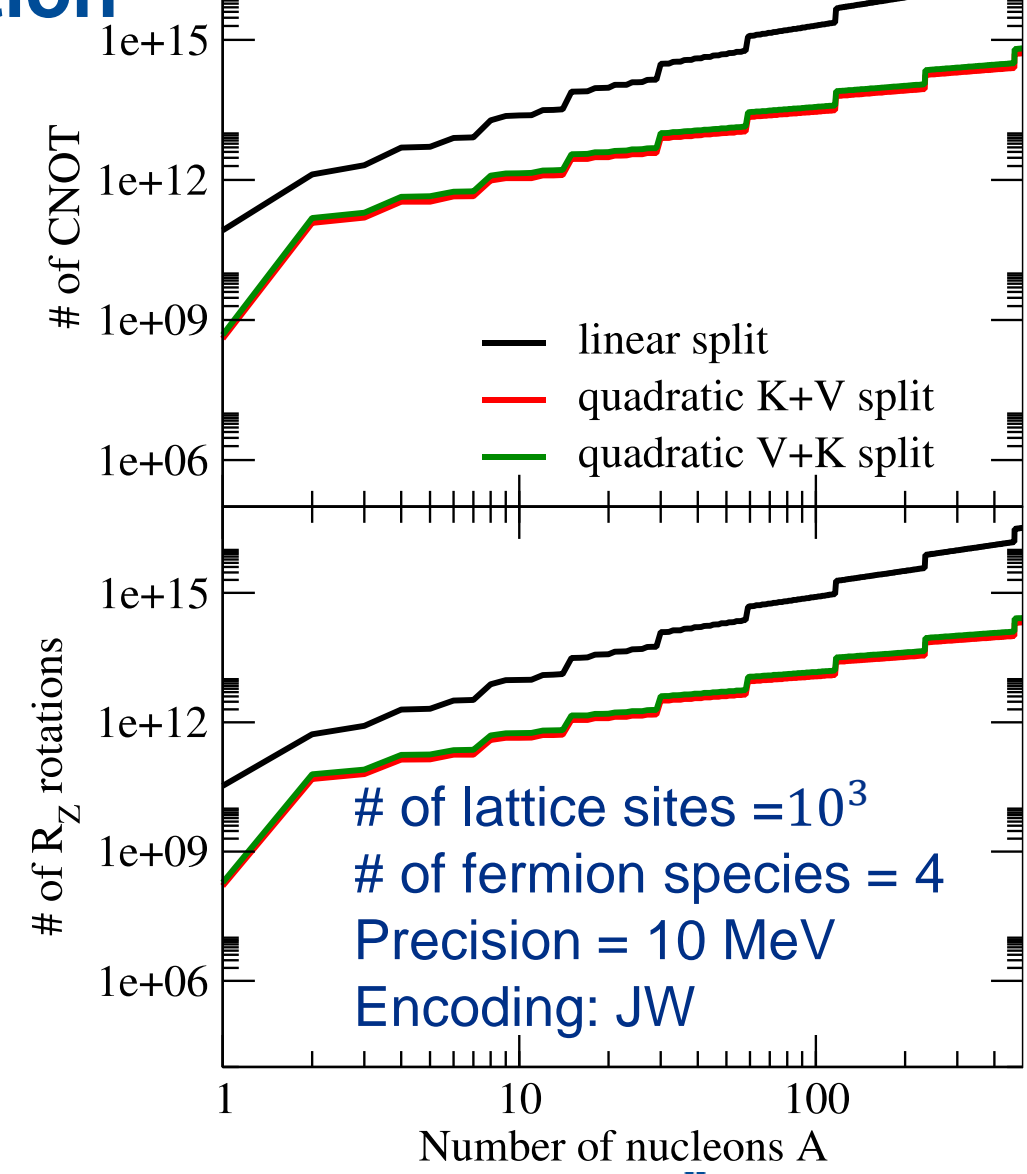




\section{NISQ implementation of modified linear response algorithm}

1. Qubit encoding: small \# of nucleons

- Lattice-location encoding

2. State preparation: $\left|\psi_{\hat{O}}\right\rangle=\frac{\hat{O}\left|\phi_{0}\right\rangle}{\sqrt{\left\langle\phi_{0}\left|\hat{O}^{\dagger} \hat{O}\right| \phi_{0}\right\rangle}}$

- Approximated low-energy state $\left|\tilde{\phi}_{0}\right\rangle$ by a variational ansatz

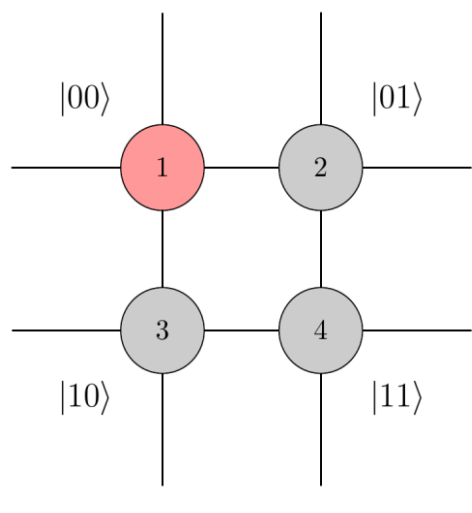

3. Quantum phase estimation of $\left|\psi_{\hat{O}}\right\rangle$ with $\widehat{U}=e^{i\left(\widehat{H}-E_{0}\right)}$

- Time evolution by $\widehat{U}(t)=e^{i\left(\widehat{H}-E_{0}\right) t}$ on a pretrained initial state

4. Measure ancilla qubits: probability distribution $\rightarrow S(\omega, \hat{O})$

- Directly measure $S(\omega, \hat{O})=\int d t\left\langle\phi_{0}\left|\hat{O}^{\dagger} e^{-i\left(\hat{H}-E_{0}-\omega\right) t} \hat{O}\right| \phi_{0}\right\rangle \quad$ (no ancilla qubits) 


\section{4-qubit proof-of-principle experiment}

- Triton toy model:

- 3 nucleons with one chosen to be static on a 2 by 2 lattice

$$
H=2 D t A-t \sum_{f=1}^{N_{f}} \sum_{\langle i, j\rangle}^{M}\left[c_{i, f}^{\dagger} c_{j, f}+c_{i, f}^{\dagger} c_{j, f}\right]
$$

- 2 effective nucleons $\left(A=2, N_{f}=2, M=4\right)$

- Two-nucleon dynamics incorporates important information about nuclear response (arXiv:1909.06400)

$$
\begin{aligned}
& +\frac{1}{2} C_{0} \sum_{f \neq f^{\prime}}^{N_{f}} \sum_{i=1}^{M} n_{i, f} n_{i, f^{\prime}} \\
& +\frac{D_{0}}{6} \sum_{f \neq f^{\prime} \neq f^{\prime \prime}}^{N_{f}} \sum_{i=1}^{M} n_{i, f} n_{i, f^{\prime}} n_{i, f^{\prime \prime}},
\end{aligned}
$$

IBMQ Poughkeepsie

- Lattice-location encoding: $A \log M=4$ qubits

- In comparison, JW needs $N_{f} M=8$ qubits

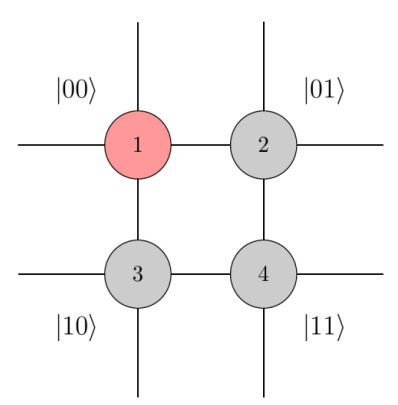

$$
\begin{gathered}
H=8 t+\frac{U}{2}-2 t \sum_{k=1}^{4} X_{k} \\
-\frac{U}{4}\left(Z_{1} Z_{4}+Z_{2} Z_{3}\right)-\frac{U}{4} \sum_{i<j<k} Z_{i} Z_{j} Z_{k} \\
C_{0}=U, D_{0}=-4 U
\end{gathered}
$$

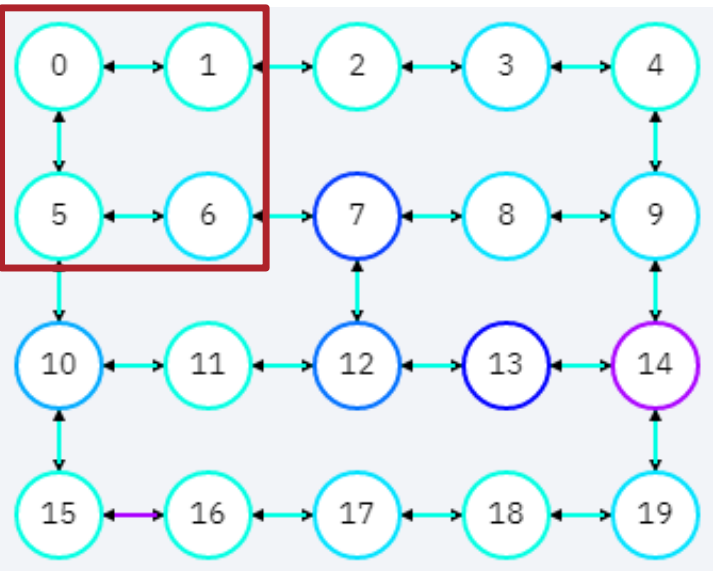




\section{State preparation with a variational ansatz}

- 2-parameter variational ansatz $|\phi(\vec{\theta})\rangle$

- Trained by a noiseless simulator to minimized the energy $E(\vec{\theta})=\langle\phi(\vec{\theta})|H| \phi(\vec{\theta})\rangle$

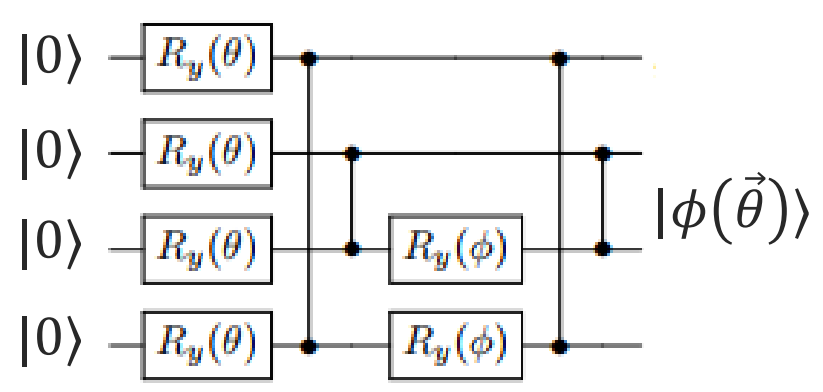

- Optimized state: $\left|\tilde{\phi}_{0}\right\rangle=\hat{O}\left|\phi_{0}\right\rangle$ (low-energy state)

- Run the pretrained circuit on the IBM QPU

\begin{tabular}{c|c} 
& Energy \\
\hline exact g.s. & -4.843 \\
simulator & -4.415 \\
\hline QPU corr & $-4.4187(98)$
\end{tabular}

- QPU shows a promising result with error mitigation (readout error mitigation and noise extrapolation) 


\section{Time evolution with 1 Trotter step}

- $1^{\text {st }}$ order Trotter's step: $U(\tau)=e^{-i \tau K} e^{-i \tau V}$

$$
\begin{gathered}
H=8 t+\frac{U}{2}-2 t \sum_{k=1}^{4} X_{k} K \\
V-\frac{U}{4}\left(Z_{1} Z_{4}+Z_{2} Z_{3}\right)-\frac{U}{4} \sum_{i<j<k} Z_{i} Z_{j} Z_{k}
\end{gathered}
$$

- Initial state: pretrained state $\left|\tilde{\phi}_{0}\right\rangle$

- 3-body contact with : $C_{3}(\tau)=\left|\left\langle 0000 \mid U(\tau) \tilde{\phi}_{0}\right\rangle\right|^{2}$ |0000): all nucleons at site 1
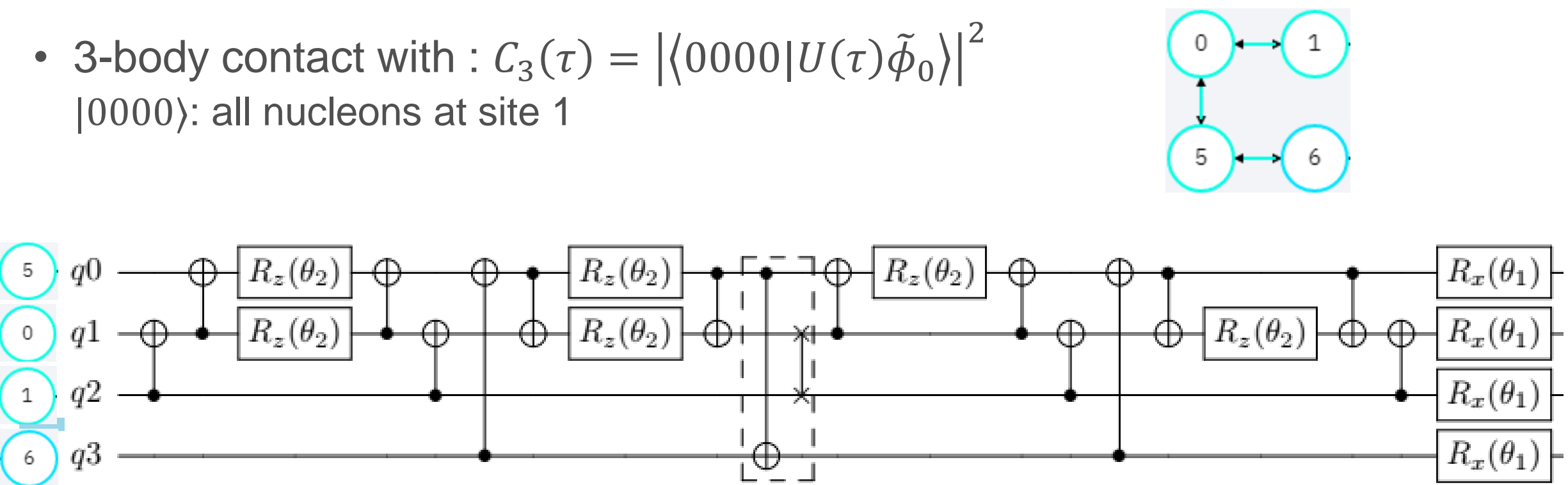


\section{Result of 1-Trotter-step time evolution}

- Expt. result: 3-week-window collection

- Output: considerable change from run to run

- error is noticeable for a single Trotter's step

$\rightarrow$ cannot do multiple Trotter's steps

- Error mitigation is insufficient to bring down the error

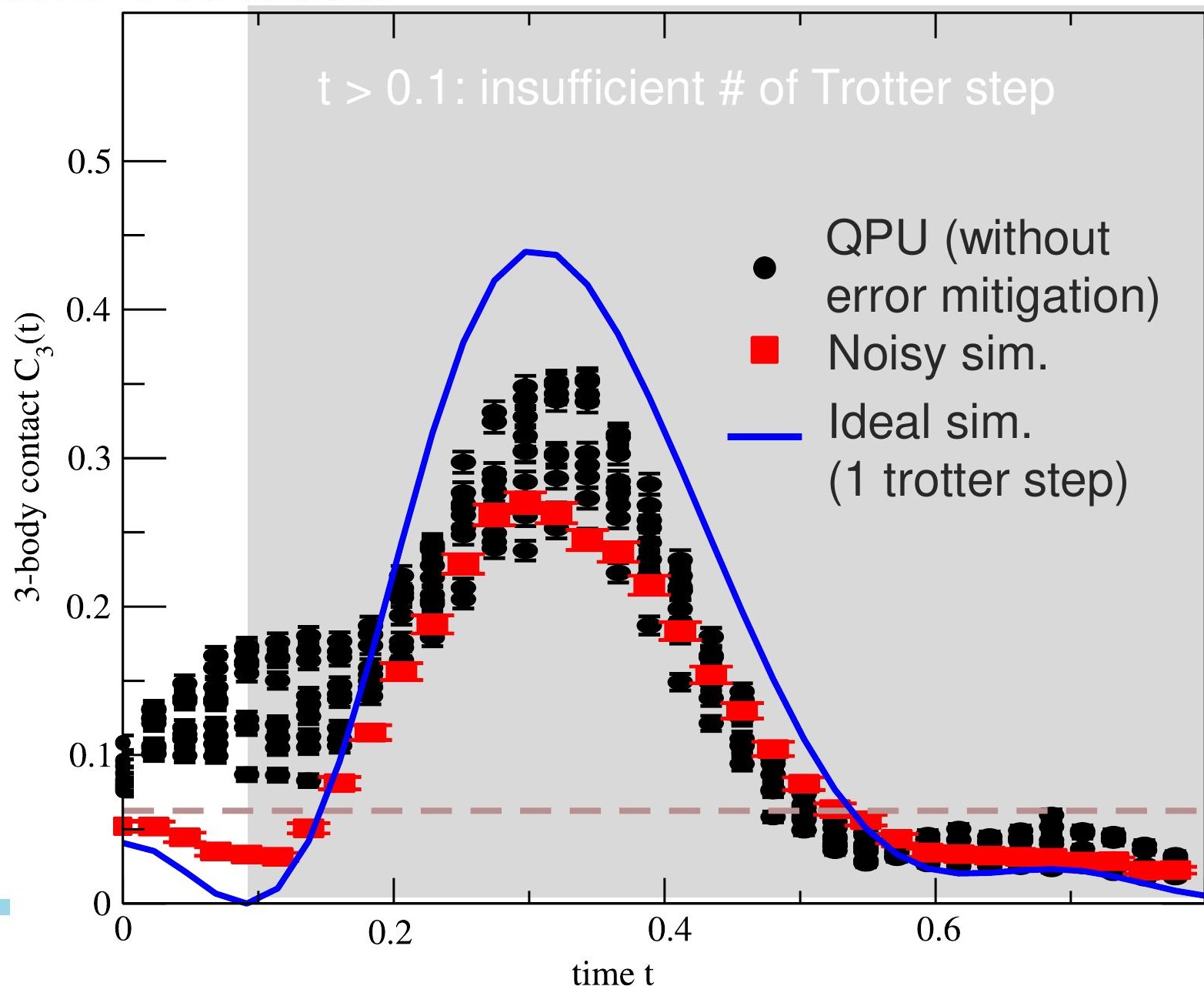




\section{Promising result and further studies needed}

1. Qubit encoding: small \# of nucleons

- Lattice-location encoding

2. State preparation: $\left|\psi_{\hat{O}}\right\rangle=\frac{\hat{O}\left|\phi_{0}\right\rangle}{\sqrt{\left\langle\phi_{0}\left|\hat{O}^{\dagger} \hat{O}\right| \phi_{0}\right\rangle}}$

- Approximated low-energy state $\left|\tilde{\phi}_{0}\right\rangle$ by a variational ansatz

3. Quantum phase estimation of $\left|\psi_{\hat{O}}\right\rangle$ with $\widehat{U}=e^{i\left(\widehat{H}-E_{0}\right)}$

- Time evolution by $\widehat{U}(t)=e^{i\left(\widehat{H}-E_{0}\right) t}$ on a pretrained initial state Further studies on error mitigation,

4. Measure ancilla qubits: probability distribution $\rightarrow S(\omega, \hat{O})$

- Directly measure $S(\omega, \hat{O})=\int d t\left\langle\phi_{0}\left|\hat{O}^{\dagger} e^{-i\left(\widehat{H}-E_{0}-\omega\right) t} \hat{O}\right| \phi_{0}\right\rangle$

hardware improvement 


\section{Overview}

- Quantum algorithm for dynamic linear response $S(\omega, \hat{O})$

- Inclusive/exclusive cross sections of neutrino-nucleus scattering

- Components: state preparation and quantum phase estimation

- Full scale studies with realistic model: potentially an important application of error-corrected quantum computer

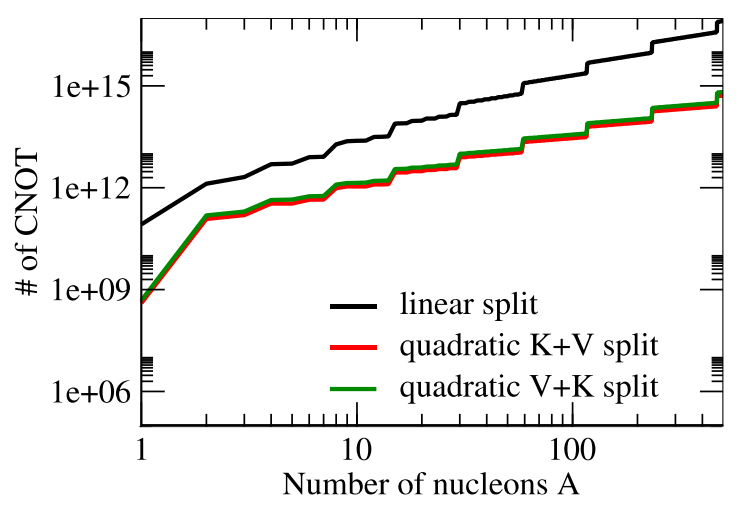

- NISQ implementation

- Components: ground state preparation and time evolution

- Promising result with today hardware

- Linear response of simple models: near-term applications with error mitigation strategies implemented and hardware improvement

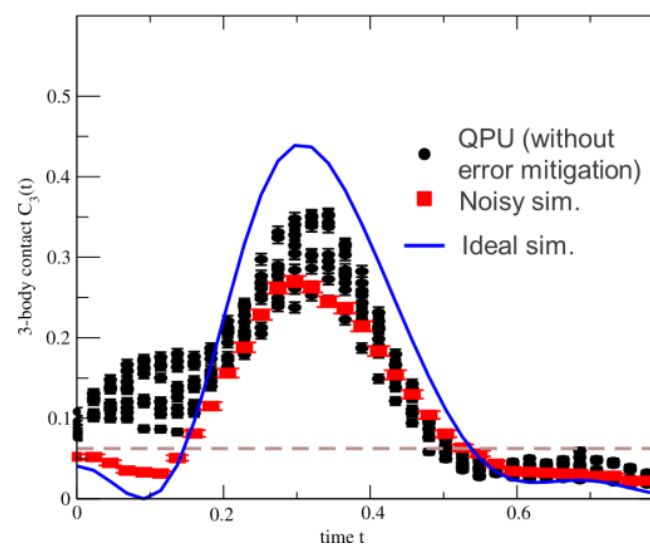

